\title{
Klumpfuss controls FMRFamide expression by enabling BMP signaling within the NB 5-6 lineage
}

María Losada-Pérez ${ }^{1}$, Hugo Gabilondo ${ }^{1}$, Isabel Molina ${ }^{1}$, Enrique Turiegano ${ }^{1}$, Laura Torroja $^{1}$, Stefan Thor $^{2}$ and Jonathan Benito-Sipos ${ }^{1, *}$

1) Departamento de Biología, Universidad Autónoma de Madrid, Cantoblanco, E 28049 Madrid, SPAIN. 2) Department of Clinical and Experimental Medicine, Linköping University, SE-581 85 Linköping, SWEDEN.

(*) Author for correspondence (JBS) (e-mail: jonathan.benito@uam.es)

Total character count: 6656

Figures: 5

Supplemental Figures: 3

RUNNING TITLE

Novel role of klumpfuss

Correspondence (J. B-S.): C/ Darwin, 2. Edificio de Biología.Unidad Fisiología Animal. Universidad Autónoma de Madrid. Campus de Cantoblanco. 28049 Madrid (Spain).

Phone: +34 914972818; Fax: +34 914978344; e-mail: jonathan.benito@uam.es 


\section{Summary}

Several broadly expressed transcription factors, expressed within most, if not all, embryonic NB lineages, participate in neural sub-type specification. Some of these have been extensively studied in several NB lineages (e.g. components of the temporal gene cascade). However, others have been only analyzed within specific NB lineages. Hereafter, it remains elusive to what extent their functions operate in other lineages. Klumpfuss (Klu), the Drosophila ortholog of the mammalian Wilms tumor 1 (WT1) protein, is one such transcription factors. Studies in the embryonic NB4-2 lineage suggested that Klu, functions within this embryonic neuroblast lineage to ensure that two GMCs in this lineage acquires a different fate. However, due to limited lineage markers available, these observations were made only for the NB4-2 lineage. Recent findings reveal that Klumpfuss is necessary for larval neuroblasts growth and selfrenewal. To gain further knowledge about this transcription factor we have extended the study of Klu to the well-known embryonic NB5-6T lineage. In this manner, we describe a novel role for Klu in Drosophila embryonic central nervous system. Our results demonstrate that Klu is expressed specifically in the postmitotic Ap4/FMRFa neuron, where is promoting its differentiation through the initiation of the BMP signaling. Our findings indicate a pleiotropic function of Klu in the Ap cluster specification in general and particularly in the Ap4 neuron differentiation, indicating that the Klu transcription factor is a multitasking factor. Finally, our studies also indicate that a transitory downregulation of $k l u$ is crucial for the specification of the Ap4/FMRFa neuron. Similar to its mammalian ortholog WT1, $k l u$ seems to have either self-renewal or differentiation promoting functions, depending on the developmental context.

\section{KEY WORDS}

Drosophila, klumpfuss, terminal differentiation, BMP signaling, neuropeptidergic cell identity, FMRFa 


\section{Introduction}

The nervous system contains intimidating numbers of cells and vast diversity in cell types. Neurons differ from each other in many ways, including in their morphology, as well as in the type of neurotransmitters/neuropeptides, receptors and ion channels they express. Each neuronal sub-type furthermore needs to be generated at the correct place, precise time, and in accurate numbers. The combined effect of vast numbers, great diversity and daunting fidelity constitutes the very basis for the enormously complex functions of the nervous system, such as homeostasis, learning/memory and behavior. Therefore, understanding neuronal sub-type specification continues to be one of the fundamental challenges in neurobiology.

Drosophila embryonic ventral nerve cord (VNC) is an important model system for addressing basic mechanisms of nervous system development. It is becoming increasingly clear that most developmental mechanisms are highly conserved across the animal kingdom, and findings in less complex models have been crucial for elucidating the molecular and genetic mechanisms that control nervous system development in higher animals.

To generate neural diversity at the precise time and place, while establishing the correct connections, Drosophila embryonic developing VNC needs to orchestrate expression of large numbers of regulatory genes with great temporal and spatial precision. The involvement in neural sub-type specification of a set of genes encoding transcription factors that are expressed within most, if not all, embryonic NB lineages, has been broadly described. Some of these genes are components of the well-known temporal gene cascade, which controls temporal competence changes within NB lineages, generating different cell types at different time points. Nevertheless, the function of other widespread transcription factors have been only described within specific NB lineages. Hence, it remains unknown to what extent their functions operate in other lineages. Klumpfuss (Klu) is one such transcription factors. $k l u$ encodes a protein with four zinc finger motifs of the $\mathrm{C} 2 \mathrm{H} 2$ type, three of which are homologous to those of the proteins of the EGR transcription factor family while the fourth is highly homologous to the divergent zinc finger of Wilms' tumor-associated protein (WT-1; (Klein and Campos-Ortega, 1997). Klu is expressed in almost all embryonic NBs, if not all, and it has been found in the GMC-2 of several lineages. Klu has been shown to prevent the GMC4-2b from adopting the GMC4-2a fate, by repressing even skipped (eve) 
expression in GMC4-2b; and loss of klu expression leads to a duplication of GMC4-2a fate. Although it is known that Klu does not affect the progeny of GMC4-2a, the role of Klu in later born GMCs in the NB4-2 lineage has not been examined (Klein and Campos-Ortega, 1997; McDonald et al., 2003; Yang et al., 1997). From this study it was concluded that $k l u$ functions within embryonic NB lineages to ensure that each GMC in a lineage acquires a different fate. However, due to limited lineage markers available, these observations were made only for the NB4-2 lineage. Recent findings pinpoint the importance of the transcription factor Klumpfuss (Klu) as a regulator of self-renewal in larval brain neuroblasts, whose overexpression results in the formation of transplantable brain tumors (Berger et al., 2012; Xiao et al., 2012). Klu is necessary for the maintenance of type I and II larval brain neuroblasts, as klu mutant larvae showed progressive loss of both types of neuroblasts due to premature differentiation.

We are taking a comprehensive molecular and genetic approach aimed at understanding neuronal sub-type specification, by using the Drosophila embryonic thoracic neuroblast 5-6 (NB5-6T) as a model. This NB can be readily identified by the specific expression of different ladybird-early $(K)($ lbe $(K))$ reporters (Baumgardt et al., 2009; De Graeve et al., 2004), and it is generated in each of the six thoracic VNC hemisegments. Each NB5-6T produces a mixed lineage of 20 cells (Baumgardt et al., 2009). At the end of its lineage, the NB5-6T generates directly, without a GMC intermediate, a set of four interneurons denoted "the Ap cluster", defined by expression of the LIM-homedomain transcription factor Apterous (Ap; mammalian Lhx2a-b), and the transcription cofactor Eyes absent (mammalian Eya1-4) (Lundgren et al., 2005; Miguel-Aliaga and Thor, 2004). The birth order of each Ap neuron is stereotyped, and the number of the neuron refers to its birth order. The four Ap neurons can be further subdivided into three different neuronal sub-types: the Ap1/Nplp1 and Ap4/FMRFa neurons, expressing the Nplp1 and FMRFamide neuropeptides, respectively, and two "generic" Ap cluster neurons, herein denoted Ap2 and Ap3 (Fig. 1A and B; (Baumgardt et al., 2009; Baumgardt et al., 2007) (Benveniste et al., 1998; Park et al., 2004).

To further understand the development of this lineage and the specification of the Ap neurons, we have conducted a "targeted screen" (Gabilondo et al., 2011) of genes expressed in the VNC (Brody et al., 2002) that alter the FMRFa pattern when mutated. One of the mutants identified in this screen, by its loss of FMRFa expression, was klu. Here, we identify a novel role for Klu in Drosophila embryonic central nervous system. 
Our results demonstrate that Klu is expressed specifically in the Ap4/FMRFa neuron, where it is selectively controlling the BMP signaling by the regulation of the BMP type I receptors. Our results indicate a pleiotropic function of Klu in the Ap cluster specification in general and particularly in the Ap4 neuron differentiation, demonstrating that the Klu transcription factor is a multitasking factor. Finally, our studies also indicate that a transitory downregulation of $k l u$ is pivotal for the specification of the Ap4/FMRFa neuron. 


\section{Results}

\section{The loss-of-function of klumpfuss abolishes the FMRFa expression in the Ap cluster}

To beginning dissecting the role of klu in the latter part of the NB5-6 we analyzed the terminal differentiation markers Nplp1 and FMRFa; neuropeptides expressed by Ap1 and Ap4 respectively. While the Nplp1 expression was largely unaffected in klu embryos (Fig. 1F, L, P and Q), we found an almost complete loss of FMRFa in the lateral thoracic areas (Fig 1 G, M, P and Q). Of note, the anterior SE2 FMRFa neurons are completely unaffected (Fig 1G). Since recent studies showed that loss-of-function of klu promotes a precocious neuroblasts differentiation (Berger et al., 2012; Xiao et al., 2012), we asked whether or not this putative precocious differentiation could preclude the generation of Ap neurons by the final part of the NB5-6 lineage in klu mutants. To this end we utilized the Eyes absent (Eya) and apterous-Gal4>UAS-GFP markers; specific markers for Ap neurons. In klu mutants, we observed 4 Ap neurons in over 76\% of hemisegments (Fig 1H, L-N and Q), although occasionally (14\%) less or more than 4 Ap neurons developed per hemisegment. However, this alteration was not statistically significant (Fig 1 Q). Hence, these results suggested that neuroblast lineage progression was not severily affected, and cannot explain the lack of FMRFa in the Ap4 neuron.

We next analyzed expression of the Dimmed (Dimm) basic-helix-loop-helix transcription factor; a "master gene" of the neuropeptidergic identity (Allan et al., 2005; Hamanaka et al., 2010; Hewes et al., 2003), normally expressed in both the Ap1/Nplp1 and Ap4/FMRFa neuropeptide neurons (Park et al., 2008). We found that klu mutants display a significant decrease of Dimm expression in the Ap cluster (Fig.1 N, P and Q). This reduction was explained by the existence of two Dimm phenotypic groups ( $n=72$ hemisegments): $50 \%$ of the clusters were wild type (2 Dimm cells/cluster), and 50\% had altered number of Dimm cells (5,5\% had 3 Dimm cells/cluster and 44,4\% had 1 Dimm cell/cluster). However, reduction of FMRFa was equal in both groups demonstrating that there is no correlation between FMRFa and Dimm phenotype. These results highlight that loss-of-function of klu produce a selective defect in the differentiation of the Ap4/FMRFa neuron. However, neither the neuroblast lineage progress nor the Ap1/Nplp1 specification is affected in klu embryos. 


\section{Klumpfuss is highly expressed in neuroblasts but is not rapidly downregulated during differentiation}

Previous studies showed that Klu is highly expressed in larval brain type I neuroblasts but is rapidly downregulated in GMCs. In type II lineages, Klu is expressed in the neuroblast but is lost from immature intermediate neural progenitors (INPs), reappearing in mature INPs and disappears again when the GMCs are formed (Berger et al., 2012; Xiao et al., 2012). We mapped in detail the Klu expression in the NB5-6T lineage, focusing on the end of the lineage, when the Ap cluster is generated. To this end we used an antibody specific to Klu protein (Yang et al., 1997). The NB5-6T neuroblast delaminates at late stage 8 . During stages 8 and 9 we detected weak expression of Klu (Fig 2A and L; not shown). Klu staining became stronger at stage 10, and robust expression of Klu was evident until the cell cycle exit of the NB5-6T neuroblast at stage 15 (Fig 2B-H and L). Regarding the Ap cluster (generated from late stage 12 until stage 15), we found that Klu was expressed in the newly-born Ap1-Ap3 neurons (Fig 2E-G and L). However, this expression was lost shortly afterwards. This succinct pulse of Klu expression in these newly-born Ap neurons correlates well with an inheritance of Klu protein from the neuroblast, but it is incongruent with the urgency of downregulation of Klu suggested for GMCs (reported both in type I and II lineages) and immature INPs (of type II neuroblasts) (Berger et al., 2012; Xiao et al., 2012).

However, the Klu expression profile in Ap4/FMRFa was different. In Ap4 we also observed Klu expression in the newly-born neuron (stage 15; Fig $2 \mathrm{H}$ and L), but in this case robust Klu staining was detected until the end of the stage 17 (Fig 2I-J and L) in the Ap4/FMRFa cell. Finally, at stage 18hAEL, expression of Klu was no longer evident in the NB5-6T lineage including the Ap cluster. Similarly, Klu expression was lost from the rest of the VNC (Fig $2 \mathrm{~K}$ and L; not shown).

\section{Klumpfuss overexpression cannot induce dedifferentiation in mature Ap neurons}

The $k l u$ mutant analysis indicated that loss-of-function of $k l u$ does not lead into a premature differentiation of the NB5-6 lineage. It has been previously reported that Klu overexpression causes dedifferentiation of immature INPs within larval Type-II lineages (Berger et al., 2012; Xiao et al., 2012). However, no detectable phenotype was found in larval type I NBs. We therefore asked whether or not overexpression of klu could cause dedifferentiation of the mature Ap neurons. 
To test this notion, we overexpressed $k l u$ from different drivers, all of which maintain expression in the post-mitotic Ap1-4 cells up to 18h AEL stage: worniu-Gal4, prosperoGal4, elav-Gal4 (Fig. 3C-D and E-G), and apterous-Gal4 (which has previously been shown to direct expression to all four post-mitotic cells, including the Ap4 neuron; Fig. 3C and H;(O'Keefe et al., 1998). Of note, the four Ap neurons were generated, and their number was largely unaffected, in all genetic combinations of klu overexpression (Fig. 3A; not shown). To determine whether or not they retain their terminal differentiation markers we studied the expression of Dimm, Nplp1 and FMRFa. Dimm and Nplp1positive neurons were numerically unaffected in those genetic backgrounds (Fig. 3A and D). However, overexpression of $k l u$, similar to its loss-of-function, produces a selectively lack of FMRFa in the Ap4 neuron (Fig. 3D-H). Hence, this set of results indicate that FMRFa expression is specifically and completely lost from all Ap4 neurons when klu expression is increased and/or maintain longer than normal, while other typical markers of the mature postmitotic Ap neurons (Eya, Dimm and Nplp1) remain intact. We concluded that klu overexpression is not able to induce dedifferentiation in Ap neurons.

Klu is expressed in the newly-born Ap4 cell. However, its expression disappears at late Stage 17. Together with the overexpression data, this led us to think that klu may need to be downregulated in the Ap4 neuron for its proper specification. To address this hypothesis, we misexpressed klu using late drivers: dimm-Gal4 (which directs expression into late post-mitotic cells, when they acquire the neuropeptidergic fate; Fig. 3C; (Hewes et al., 2003)) and fmrfa-Gal4 (a late driver directing expression by the regulatory sequence of the fmrfa gene;(Suster et al., 2003)). Hence, both of them are expressed when the Ap4 neuron is almost completely specified. The misexpression of $k l u$ from the dimm-Gal4 driver allowed a partial expression of FMRFa (31\%; Fig. 3D and I), and the klu misexpression from the fmrfa-Gal4 did not interfere at all with the FMRFa expression (100\%; Fig 3D and J). Altogether, these observations indicate that there is a transient and critical time window in which klu needs to be downregulated for the expression of the FMRFa neuropeptide in the Ap4 neuron but not in the SE2 neurons.

\section{Klumpufss is not regulating Apterous neuron determinants}

To unravel the role of Klu in the Ap window in more detail, we analyzed expression of a number of other genes crucial for proper Ap neuron specification. These included the 
temporal genes castor (cas; mammalian casz 1) and grainy head (grh; mammalian grainyhead [mgr]), as well as the sub-temporal genes squeeze (sqz; mammalian CIZ), nab (mammalian Nabl-2) and seven up (svp; mammalian COUP-TFI andII)(Baumgardt et al., 2009; Benito-Sipos et al., 2011; Terriente Felix et al., 2007).

We observed no altered expression of these temporal and sub-temporal factors (Fig. S3A-E, G-H and M-N) in klu embryos. Thus, the temporal progression in the latter part of NB5-6T development is not affected in klu mutants. Next, we analysed the expression of the Ap neuron determinant collier/knot (col) (mammalian ebfl3;(Baumgardt et al., 2009)). We found that Col expression was numerically unaffected in $k l u$ mutants (Fig. S3 L-N). Summarizing, we conclude that klu is not regulating the Ap neuron determinants cas, grh, sqz, nab, svp and col.

\section{BMP signaling is interrupted in klumpfuss mutants}

At this point, we had analysed in klu mutants most of the genes which have been identified as crucial for the specification of the Ap4/FMRFa cell fate. None of them were altered in klu mutants. Thus, if Ap4 determinants are not affected, why is FMRFa lost in klu mutants?

FMRFa expression in the Ap4 neuron is also critically dependent on a retrograde instructive signal; the target-derived transforming growth factor-b (TGF-b)/bone morphogenetic protein (BMP;(Allan et al., 2003; Marques et al., 2003). The Ap4 neuron innervates a peripheral secretory gland, the dorsal neurohemal organ (DNH), where it receives the TGF-b/BMP ligand Glass bottom boat (Gbb), which finally triggers expression of the FMRFa neuropeptide gene. In Drosophila, BMP signaling leads to the phosphorylation and nuclear translocation of the Smad protein Mothers against dpp (Mad), which can be monitored using antibodies specific to phosphorylated Mad (pMad;(Dorfman and Shilo, 2001; Marques et al., 2003; Tanimoto et al., 2000)). Targetderived BMP signaling is also known to occur in most, if not all, motoneurons in the VNC (Aberle et al., 2002). Hence, we used a specific antibody to pMad to assay for BMP activation in Ap4 neurons in a klu mutant background. While there was no obvious loss of pMad staining in the VNC in general (data not shown), we found a prevalent loss of pMad expression in the Ap4 neuron (Fig 4B and E). In addition, absence of FMRFa was always associated with an absence of Ap4 pMad staining (Fig. 
4B; $100 \%$ ). Of note, the lack of FMRFa in the overexpression of klu is not explained by the absence of pMad (Fig. S1).

Why is BMP signaling interrupted in klu mutants? The loss of pMad could reflect an absence of the target gland, the DNH itself. To address this issue, we revealed the DNH by the btn-lacZ expression (Allan et al., 2003), and we found an apparent normal DNH in klu mutants (Fig. 4D). Another possibility is a failure of the Ap4/FMRFa neuron to project its axon to the $\mathrm{DNH}$, with an accompanying failure to receive the TGF-b/BMP ligand Gbb. To explore this possibility, we analyzed and quantified the innervation of the Ap4/FMRFa neuron in klu mutants, but no significant statistical differences were found between controls and klu mutants in the DNH innervation (Fig. 4D and F).

Since the Ap4/FMRFa neuron properly innervates its target gland in klu mutants, we wanted to address a possible role for $k l u$ in the specification of the DNH itself, which could result in the absence of the ligand Gbb in this gland. Although we did not observe Klu expression in the DNH (Fig. 4G) it is possible that klu plays an early role in DNH specification. To circumvent this issue, we attempted to rescue FMRFa in klu mutants by providing $g b b$ directly to the DNH (slit-Gal4>UAS-gbb; klu mutant). However, this failed to restore FMRFa expression (Fig 4I and K). Hence, these results lead to the conclusion that the Ap4 neuron is not able to respond to the ligand Gbb. To test this notion, we provided $g b b$ in the Ap4 neuron itself, by using ap-Gal4/UAS-gbb in klu mutants (previous studies reported that the misexpression of $g b b$ rescues $g b b$ mutants cell autonomously (Allan et al., 2003)). However, we found no rescue of FMRFa in this genetic background (Fig.4J and K). Therefore, we determine that the Ap4 neuron is not competent to respond to the Gbb ligand in klu mutants.

\section{Expression of the type-I BMP receptors rescue FMRFa expression in the Ap4/FMRFa neuron}

In the Ap4/FMRFa cell the activation of the BMP pathway begins when the ligand Gbb binds to a tetrameric membrane receptor complex (consisting of two receptor pairs type I and type II BMP receptors)(Allan et al., 2003). Then, the constitutively active type II BMP receptors (wishful thinking - wit) recruit and then phosphorylate their type I BMP partners (saxophone (sax)- and thick veins $(t k v)$ ). The type-I receptors in turn phosphorylate the cytoplasmic receptor-regulated Smad (R-Smad) Mad. The R-Smad 
Mad then associates with common non-phosphorylated Smads (co-Smads), Medea in

Drosophila, to form a "phospho-Mad" complex (pMad) that translocates to the nucleus to participate in transcriptional regulation (Fig. 5A)(reviewed in(Keshishian and Kim, 2004)).

Why then is the Ap4 neuron not able to respond to the Gbb ligand in klu mutants? To address this issue, we asked whether or not the constitutive activation of the BMP pathway is able to rescue the FMRFa expression in klu mutants. In Drosophila, constitutive activation of the BMP pathway can be achieved by expression of activated versions of either one of the type I receptors sax or tkv (UAS-saxA and $U A S-t k v A$;(Haerry et al., 1998)). Is the klu mutant Ap4 neuron able to transduce the signal from activated type-I BMP receptors? Using elav-Gal4, we expressed these modified receptors, and we found that it resulted in a 68\% rescue of FMRFa (Fig. 5B and D). We also observed ectopic FMRFa-expressing cells in the Ap cluster. These results indicate that $k l u$ mutant Ap4/FMRFa neurons are defective in their response to the Gbb ligand.

To initiate downstream responses, the type-I BMP receptors need to be recruited and phosphorylated by the type-II receptor Wit, and this phosphorylation step is activated by binding of the BMP ligand to the type-I BMP receptors in the receptor complex. Therefore, expression of constitutively active forms of the type-I BMP receptors Sax and Tkv bypasses the need for the type-II receptor Wit, while expression of normal forms of these receptors does not. Therefore, we wished to determine whether the $k l u$ phenotype is due to type-I or type-II BMP receptors. To test the involvement of the type-II receptor wit, we expressed the wild type versions of the type-I BMP receptors (elav>sax,tkv; klu mutant). Even in this scenario we found a similar rescue of FMRFa expression in klu mutants (66\%; Fig. 5C and D), demonstrating that the type-I but not the type-II receptors are at the core of the klu phenotype in Ap/FMRFa neuron. To further test this notion, we attempted to rescue the FMRFa expression in klu mutants by expressing the type II receptor Wit (elavG4>UAS-wit; klu mutants). However, we found no statistically significant rescue in this genetic background (Fig. 5D). Thus, our results indicate that $k l u$ controls, directly or indirectly, the levels or responsiveness of the plasma membrane bound type-I BMP receptors in the Ap4/FMRFa neuron. 


\section{None of the known Ap neuron determinants is controlling Klumpfuss expression}

Finally, we addressed the activation of klu at the very end of the NB5-6T lineage, when the Ap4/FMRFa neuron is generated. Given that we cannot rule out that the Ap4 neuron expresses Klu due to an inheritance from the neuroblast, we examined Klu expression both within the neuroblast at stage late 14 (when the neuroblast is generating the Ap4 cell) and within the whole Ap cluster. In those mutants where the Ap and Eya markers are lost, we used the ladybird-early $(K)$ reporter, and we identified the Ap cluster by position. Mutants for the cas, $n a b$ and $s q z$ temporal/sub-temporal genes had no apparent effect upon Klu expression (Fig S2A-B, F-I and Q-S). grh and svp mutants display an increase in the number of Ap neurons (Baumgardt et al., 2009; Benito-Sipos et al., 2011), and accordingly we found an increase of the Ap neurons expressing Klu at stage 16 (Fig S2D, K and Q-S). However, Klu expression was properly turned off at stage 18h AEL in both mutants (Fig S2E, L and Q-S). Hence, we concluded that the increase in Klu-positive neurons displayed by grh and $s v p$ embryos was merely reflecting the increase in the number of Ap neurons. Finally, we studied Klu expression in mutants for the col, ap and eya determinants, but observed no apparent global effect upon Klu expression in those mutant backgrounds (Fig S2M-S). Therefore, none of the reported Ap neuron determinants is controlling Klu expression, and the factor/s involve in the Klu activation remain/s elusive. 


\section{Discussion}

We find that Klu is expressed in the newly-born Ap4/FMRFa neuron and this expression is detected until the end of the stage 17. Our results demonstrate that the critical role of Klu in this scenario is the control of the type-I BMP receptors expression. Nevertheless, our findings indicate a pleiotropic function of Klu in the Ap cluster specification in general and particularly in the Ap4 neuron differentiation, indicating that the Klu transcription factor is a multitasking factor. Finally, we have found that a transient suppression of $k l u$ is critical for the specification of the Ap4/FMRFa neuron. This set of results unravels a new and non-canonical role of the transcription factor Klu in the neural cell specification.

\section{A new role of Klumpfuss in neural cell specification}

Previous studies carried out in the NB4-2 lineage reported that Klu is preventing the second-born daughter cell in that lineage (GMC4-2b) from adopting the GMC4-2a fate. This is achieved by repressing even skipped (eve) expression in GMC4-2b, and loss of $k l u$ expression leads to a duplication of GMC4-2a fate. However, due to limited lineage markers available, the role of Klu both in later born GMCs in the NB4-2 lineage and other NB lineages has not been examined (Klein and Campos-Ortega, 1997; McDonald et al., 2003; Yang et al., 1997). Recent studies in larvae pinpoint the transcription factor Klu as distinguishing a type II neuroblast from an intermediate neural progenitor (INP) in larval brains. Klu functions to maintain the identity of type II neuroblasts, and klu mutant larval brains show progressive loss of type II neuroblasts due to premature differentiation (Xiao et al., 2012). Additionally, studies from sensory organ precursors (SOPs) suggest a similar mechanism of action: over-expression of Klu results in formation of supernumerary bristles, whereas loss of its function leads to loss of bristles due to the lack of determination of the corresponding SOP (Kaspar et al., 2008). Hence, all previous data around Klu indicate that this transcription factor distinguishes between two fates ("A" versus "B"). In all these cases the loss-of-function of $k l u$ gives extra cells with identity "A", and the gain-of-function of klu gives extra cells with identity "B". However, our studies of NB5-6 reveals a different role for Klu. Loss and gain-offunction of klu does not merely lead to confusion with an incorrect neuronal identity. By contrast, Klu is necessary for the proper initiation of one of the components of the combinatorial code necessary for the Ap4/FMRFa specification: the BMP signaling. 
Importantly, neither BMP signaling nor Klu is sufficient to activate the FMRFa expression in other Ap neurons. Therefore, our results highlight a new function of the transcription factor Klu in neural cell specification.

\section{Klu, as its mammalian ortholog WT1, have either self-renewal and differentiation functions during development, depending on context}

Klu encodes a transcriptional regulator characterized by four zinc-finger motifs in the C-terminus, and it is the fly ortholog of the mammalian Wilm's tumor 1 (WT1) gene (Klein and Campos-Ortega, 1997; Yang et al., 1997). Paradoxical and contradictory functions have been described around WT1, which can act as transcriptional activator or repressor, promoting proliferation, differentiation or apoptosis, in a highly contextdependent manner. In mammalians, mutations in WT1 results in tumor formation, and WT1 has also been found necessary for the proliferation of certain neuronal progenitors (reviewed in(Hohenstein and Hastie, 2006; Roberts, 2005), which is in line with the reported role of Klu in self-renewal. By contrast, WT1 has also been identified in differentiation process: promoting an essential role in nephron progenitor differentiation during renal development (reviewed in(Hohenstein and Hastie, 2006; Roberts, 2005) or participating in the differentiation of the olfactory epithelium (Wagner et al., 2005), which is in agreement with the role of Klu reported here. Therefore, our findings indicate that Klu, as WT1, have either self-renewal and differentiation functions during development, depending on context.

\section{Klumpufss is required for the onset, but not for the maintenance, of the BMP signaling within the Ap4 neuron}

We have employed a number of experiments involving different markers, mutant combinations and a detailed analysis of Klu with respect to the FMRFa phenotype within the Ap cluster. Our findings reveal that at the heart of the klu phenotype in the Ap4/FMRFa neuron lays defects in the BMP signaling. Previous studies reported that FMRFa expression is maintained by persistent retrograde BMP signaling in the Ap4 neuron (Eade and Allan, 2009). Here, we find that klu is a crucial regulator of the BMP

signaling. However, Klu expression is extinguished at the end of stage 17. Hence, Klu is necessary for the initiation of BMP signaling, but is dispensable for its maintenance within the Ap4 neuron. Further investigation will be necessary to elucidate the molecular function of $k l u$ in controlling the expression of the type-I BMP receptors. 
Is the control of BMP signaling a global/conserved function of Klu? klu mutants do not display a general absence of pMad staining within the whole VNC (data not shown). Hence, although we cannot rule out a global control of the type-I BMP receptor by $k l u$, its role seems to be highly context-dependent. Additionally, previous studies by ChIP-chip in embryonic mouse kidney tissue identified transcriptional targets of WT1, the putative mammalian ortholog of Klu, in nephron progenitor cells during renal development in vivo. Among those targets they found several components of the BMP signaling pathway: BMP4, BMP7, the two R-Smads Smad3 and Smad4, and the two innibitory I-Smads Smad6 and Smad7. However, none of the BMP receptors were found in those studies. Hence, although WT1 has been related with the control of BMP signaling, the mechanisms underlying this control seem to be multiple and highly cellspecific. Intriguingly, in those studies, Nab1 and Nab2, the mammalian orthologous of the sub-temporal factor Nab, were also found as targets of WT-1. Here we have studied $\mathrm{Nab}$ expression in loss-of-function of klu, and Nab expression was unaffected in that mutant background 


\section{EXPERIMENTAL PROCEDURES}

\section{Fly Stocks}

Fly stocks were raised and crosses were performed at $25^{\circ} \mathrm{C}$ on standard medium. The following fly mutant alleles were used: $s q z^{i e}$ (Allan et al., 2003); $a p^{m d 544}$ (referred to as ap-Gal4;(O'Keefe et al., 1998); $\operatorname{cas}^{41}$ (Mellerick et al., 1992); $\operatorname{grh}^{I M} ; k^{212 I R 51 C}$ (Cheah et al., 2000); Yang, 1997 \#129\} (provided by W. Chia); col $^{1} /$ col $^{3}$ (Baumgardt et al., 2007); $n a b^{S H 143}, n a b^{R 52}$ (provided by Fernando Díaz-Benjumea) ; eya ${ }^{C l i-I D}$; $s v p^{l}$ (Kanai et al., 2005); $g s b^{01155}$ (referred to as gsb-lacZ); elav-Gal4; $D f(2 R) P c l 7 B$ (referred to as grh $^{D f}$ ); prospero-Gal4;worniu-Gal4; dimm-Gal4; fmrfa-Gal4 (provided by P. Taghert); btn-lacZ; slit-Gal4 (provided by Christian Klämbt); UAS-gbb, UAS-sax ${ }^{A}, U A S-t k v^{A}$, $U A S$-sax, UAS-tkv (provided by M. O'conor); lbe(K)-Gal4; UAS- nmEGFP (Baumgardt et al., 2009). Mutants were kept over CyO, Act-GFP; CyO, Dfd-EYFP; TM3, Ser, ActGFP; CyO, twi-Gal4, UAS-GFP; TM3, Sb, Ser, twi-Gal4, UAS-GFP; or TM6, Sb, Tb, $D f d-E Y F P$ balancer chromosomes. As wild type, Oregon- $R$ was often used. Unless otherwise stated, flies were obtained from the Bloomington Drosophila Stock Center.

\section{Immunohistochemistry}

The antibodies used were: rabbit $\alpha$-Klumpfuss (1:1,000) (provided by X.H.Yang) (Yang et al., 1997). Guinea pig $\alpha$-Col $(1: 1,000)$, guinea pig $\alpha$-Dimm $(1: 1,000)$, chicken $\alpha$ proNplp1 $(1: 1,000)$, rabbit $\alpha$-proFMRFa (1:1,000) (Baumgardt et al., 2007); mouse $\alpha$ Seven up (1:50) (a gift of Y.Hiromi); rabbit $\alpha$-pMad (1:200) (41D10, Cell Signaling); mAb $\alpha$-Eya10H6 (1:250) (from Developmental Studies Hybridoma Bank, Iowa City, IA, US). All polyclonal sera were pre-absorbed against pools of early embryos.

Secondary antibodies were conjugated with FITC, Rhodamine-RedX or Cy5, and used at 1:500 (Jackson ImmunoResearch, PA, US). Embryos were dissected in PBS, fixed for 25 minutes in 4\% PFA, blocked and processed with antibodies in PBS with $0.2 \%$ Triton-X100 and 4\% donkey serum. Slides were mounted with Vectashield (Vector, Burlingame, CA, US). In all cases wild-type and mutant embryos were stained and analyzed on the same slide.

\section{Confocal Imaging, Data Acquisition and Staining quantification}

A Zeiss META 510 Confocal microscope was used to collect data for all fluorescent images; confocal stacks were merged using LSM software or Adobe Photoshop CS4. 
Where appropriate, images were false colored to facilitate for color-blind readers or to facilitate the understanding of the paper.

\section{Statistical Methods}

Statistical calculations were performed using SPSS software (v15.0.1). To address statistical significance, Student's t-test or, in the case of non-Gaussian distribution of variables, a nonparametric Mann-Whitney U test was used. Images and graphs were compiled in Adobe Illustrator.

\section{ACKNOWLEDGMENTS}

We are grateful to M. Pita, M. O'conor, C. Klämbt , P. Taghert, W.Chia, M. Baumgardt, J. Skeath, W. Odenwald, T. Isshiki the Developmental Studies Hybridoma Bank at the University of Iowa, and The Bloomington Stock Center for sharing antibodies and fly lines. We also want to thank to Carlos Sánchez, Verónica Labrador and Angeles Muñoz for their technical assistance in confocal microscopy. This work was supported by a grant from the Spanish Ministerio de Ciencia e Innovación (BFU-2008-04683-C02-02) to L.T and by the Swedish Research Council, by the Swedish Strategic Research Foundation, by the Knut and Alice Wallenberg foundation, by the Swedish Brain Foundation, by the Swedish Cancer Foundation, by the Swedish Royal Academy of Sciences to ST. 


\section{FIGURE LEGENDS}

\section{Figure 1}

\section{klumpfuss is critical for Ap4/FMRFa neuron differentiation}

(A) Model of the NB 5-6T lineage based on previous studies (Baumgardt et al., 2009).

The four Ap cluster neurons are the last-born neurons, and are generated without GMC intermediate. (B) Previous reports identified several regulatory genes specifically expressed in subsets of Ap neurons, acting to specify their identities (see text for references). (C-N) Expression of the Ap cluster determinants Eya and Dimm, and of the terminal identity markers FMRFa and Nplp1, in control and klu mutants: stage 18hAEL embryonic VNCs (anterior up; brackets outlining the three thoracic segments). The observed phenotypes are summarized in the cartoons $(\mathrm{O}$ and $\mathrm{P})$, and in a graphical representation of the quantified results ( \pm S.D.) (Q): $n \geq 10$ VNC in all genotypes: asterisks (*) denote significant difference compared to control (Student t-test, $\mathrm{p}<0.001$ ).

(F and L) Nplp1 expression is not lost in the Ap clusters, as revealed by proNplp1 staining. ( $\mathrm{G}$ and $\mathrm{M})$ Staining against FMRFa shows absence of expression in klu mutants in Ap4/FMRFa neurons (brackets). (G) In contrast, FMRFa expression in the more anterior and medial SE2 neurons, generated by a different neuroblast (LosadaPerez et al., 2010), is largely unaffected in klu. (H and L-N) Expression of Eya reveals that the Ap cluster is generated in all thoracic hemisegments in klu mutants. (N and Q) Expression of Dimm is numerically reduced within the Ap cluster. Wild type and mutant VNCs were stained and analyzed on the same slide. Genotypes: (C-E, I-K) OregonR. (F-H, L-N) $k l u^{212 l R 51 C} / k^{2} u^{212 l R 51 C}$.

\section{Figure 2}

\section{Klumpfuss expression in the NB5-6T lineage}

(A-L) Expression of Klu (green) within NB5-6T, during embryonic development. To the right are side-view graphic representations of the lineage. Images are composed from confocal stacks, in $\mathrm{G}$ and $\mathrm{H}$ subdivided into two sub-stacks, from dorsal (up) to ventral (down). Anterior is up in all images. (A-D) NB5-6T is identified as the anteriorand lateral-most neuroblast within the $g s b^{\text {lacZ }}$ domain (not shown), as well as by cell size and staining for Deadpan (magenta). (E-H) Alternatively, NB5-6T is identified by reporter gene expression driven from NB5-6 specific lbe $(K)$ enhancer (magenta). Ap1, Ap2, Ap3 and Ap4 neurons are identified by position and different levels of Col (not 
shown) (Baumgardt et al., 2009). (I-K) During later stages, Ap1, Ap2, Ap3 and Ap4 neurons are identified by expression of ap-Gal4>UAS-GFP (magenta), different levels of Cas staining (not shown) (Baumgardt et al., 2009). (A-H) We detect expression of Klu in the neuroblast from stage 9 until the cell cycle exit of the NB5-6T neuroblast at stage 15. (E-K) In the Ap cluster we find that Klu is expressed in the newly-born Ap1Ap3 neurons (E-H). This expression is extinguished shortly afterwards. However, in the Ap4 neuron we also observed Klu expression in the newly-born neuron $(\mathrm{H})$, but in this case Klu staining is detected until the end of the stage $17(\mathrm{~J})$. Finally, at stage 18hAEL, expression of Klu is no longer evident neither within the Ap cluster nor in the rest of the VNC (K; not shown). (L) Cartoon depicting the expression of Klu in the NB5-6T lineage. Genotypes: (A-D) $g s b^{l a c Z} /+$. (E-H) lbe(K)-Gal4, lbe(K)-Gal4/UAS-nmEGFP. (I-K) ap-Gal4/UAS-nmEGFP /+.

\section{Figure 3}

\section{Misexpression of klumpfuss abolishes specifically the FMRFa expression in the}

\section{Ap4 neuron}

(A) Dimm expression is normal in a $k l u$ missexpression background. (B and E-J) Expression of FMRFa at 18hAEL in control (B) and klu misexpression from different drivers: (E) worniu-Gal4, (F) prospero-Gal4, (G) elav-Gal4 (all of them are expressed in the NB56T, and their expression persist in the post-mitotic Ap4 cell), and (H) apterous-Gal4 (expressed in the post-mitotic Ap neurons). In those misexpression contexts FMRFa expression is selectively abolished from the Ap4 neuron. (I-J) Expression of FMRFa at 18hAEL in klu misexpression from very late drivers: (I) dimm-Gal4 (which directs expression into late post-mitotic cells, when they acquire the neuropeptidergic fate) and (J) fmrfa-Gal4 (a late driver directing expression under the governed by the regulatory sequence of the fmrfa gene; Suster et al., 2002). See text for details. FMRFa expression is reduced in the misexpression of klu from the dimm-Gal4 driver, but is largely unaffected in the misexpression of klu from the very late driver fmrfa-Gal4. (C) Cartoon depicting the wild type expression of Klu (blue) and the timing of the expression of the drivers used in this figure (yellow). (D) Quantification of the observed phenotypes ( \pm S.D.) ( $\mathrm{n} \geq 30$ hemisegments in all genotypes; asterisks (*) denote significant difference compared to control (Chi-square test, $\mathrm{p}<0.001$ ). Wild type and mutant VNCs were stained and analyzed on the same slide. Genotypes: (A) elav-Gal4>UAS-klu. (B) OregonR. (E) 
worniu-Gal4/UAS-klu. (F) prospero-Gal4/UAS-klu. (G) elav-Gal4/UAS-klu. (H)

apterous-Gal4/UAS-klu. (I) dimm-Gal4/UAS-klu. (J) fmrfa-Gal4/UAS-klu.

\section{Figure 4}

\section{BMP signaling is interrupted in the Ap4 neurons in klumpfuss mutants}

(A-B) pMad staining at 18hAEL in control (A) and $k l u$ mutant (B). pMad staining is lost in klu mutants. (C-D) Overlap of btn-lacZ (red), apterous-Gal4>UAS-nmEGFP (green) and FMRFa (blue) at 18hAEL in control (C-) and klu mutant (D). There are no gross differences between controls and klu mutants in the DNH innervation. (E) Quantification of the observed phenotypes in pMad staining ( \pm S.D.) ( $\mathrm{n} \geq 35$ hemisegments; Chi-square test, $\mathrm{p}<0.001$ ). (F) Quantification of the innervation of the Ap4/FMRFa neuron in klu mutants (t-student test, $\mathrm{p}<0.001$ ) (G-G') Overlap of $b t n$ lacZ (red) and Klumpfuss (green) at 18hAEL. Klu staining is not detected in the DNH. (H-J) Expression of FMRFa at 18hAEL in control $(\mathrm{H})$ and $k l u$ mutants expressing directly $g b b$ to the DNH (I) and $k l u$ mutants expressing directly $g b b$ in the Ap4 neuron itself ( $\mathbf{J})$ (see genotypes below and text for details). There is no rescue of the FMRFa expression in these genetic backgrounds. (K) Quantification of the observed phenotypes ( $\mathrm{n} \geq 10$ VNCs). Wild type and mutant VNCs were stained and analyzed on the same slide.

Genotypes: (A) apterous-Gal4/UAS-nmEGFP. (B) apterous-Gal4/UAS-nmEGFP; $\mathrm{klu}^{212 l R 51 C} / \mathrm{klu}^{212 l R 51 C}$. (C) apterous-Gal4/UAS-nmEGF, btn-lacZ. (D) apterous-Gal4, btnlacZ; klu ${ }^{212 l R 51 C} / k^{2} u^{212 l R 5 l C}$. (E) btn-lacZ (G) OregonR. (H) slit-Gal4, UAS-gbb; $\mathrm{klu}^{212 l R 51 C} / \mathrm{klu}^{212 l R 51 C}$. (I) apterous-Gal4, UAS-gbb; $\mathrm{klu}^{212 l R 51 C} / \mathrm{klu}^{212 l R 5 I C}$

\section{Figure 5}

Klumpfuss is regulating the expression of the type-I BMP receptors in the Ap4/FMRFa neuron

(A) The BMP pathway in the Ap4 neuron (based on Allan et al., 2003 and Marques et al., 2003). (B-C) Expression of FMRFa at 18hAEL in klu mutants co-expressing the activated sax and $t k v$ receptors (B) and co-expressing the wild type version of sax and $t k v$ receptors (C; see genotypes below). Both genetic combinations result in rescue of FMRFa expression in klu mutants $(68,6 \%$ and $65.7 \%$, respectively; in the co-expression of activated sax and $t k v$ receptors we observed ectopic FMRFa-expressing cells in the Ap cluster, but these ectopic cell were not included in the quantification of the rescue). (D) Quantification of the observed phenotypes ( \pm S.D.) ( $\mathrm{n} \geq 10$ hemisegments; Mann- 
Whitney $\mathrm{U}$ test, $\mathrm{p}<0.001)$. Wild type and mutant VNCs were stained and analyzed on the same slide. Genotypes: (B and D) elav-Gal4/UAS-sax ${ }^{A}, U A S-t k v^{A} ; k l u^{212 l R 51 C} / k l u^{212 l R 51 C}$. (C and D) elav-Gal4/UAS-sax,UAS-tkv; klu ${ }^{212 l R 51 C} / k_{l} u^{212 l R 51 C}$. (D) Orizo2, $\mathrm{klu}^{212 l R 51 \mathrm{C}} / \mathrm{klu}^{212 l R 5 l \mathrm{C}}$, elav-Gal4/UAS-wit; $\mathrm{klu}^{212 l R 51 \mathrm{C}} / \mathrm{klu} \mathrm{LIIRSIC}^{21}$.

\section{REFERENCES}

Aberle, H., Haghighi, A. P., Fetter, R. D., McCabe, B. D., Magalhaes, T. R. and Goodman, C. S. (2002). wishful thinking encodes a BMP type II receptor that regulates synaptic growth in Drosophila. Neuron 33, 545-58.

Allan, D. W., Park, D., St Pierre, S. E., Taghert, P. H. and Thor, S. (2005). Regulators acting in combinatorial codes also act independently in single differentiating neurons. Neuron 45, 689700.

Allan, D. W., St Pierre, S. E., Miguel-Aliaga, I. and Thor, S. (2003). Specification of neuropeptide cell identity by the integration of retrograde BMP signaling and a combinatorial transcription factor code. Cell 113, 73-86.

Baumgardt, M., Karlsson, D., Terriente, J., Diaz-Benjumea, F. J. and Thor, S. (2009). Neuronal subtype specification within a lineage by opposing temporal feed-forward loops. Cell 139, 96982.

Baumgardt, M., Miguel-Aliaga, I., Karlsson, D., Ekman, H. and Thor, S. (2007). Specification of neuronal identities by feedforward combinatorial coding. PLOS Biol 5, e37.

Benito-Sipos, J., Ulvklo, C., Gabilondo, H., Baumgardt, M., Angel, A., Torroja, L. and Thor, S. (2011). Seven up acts as a temporal factor during two different stages of neuroblast 5-6 development. Development 138, 5311-20.

Benveniste, R. J., Thor, S., Thomas, J. B. and Taghert, P. H. (1998). Cell type-specific regulation of the Drosophila FMRF-NH2 neuropeptide gene by Apterous, a LIM homeodomain transcription factor. Development 125, 4757-65.

Berger, C., Harzer, H., Burkard, T. R., Steinmann, J., van der Horst, S., Laurenson, A. S., Novatchkova, M., Reichert, H. and Knoblich, J. A. (2012). FACS Purification and Transcriptome Analysis of Drosophila Neural Stem Cells Reveals a Role for Klumpfuss in Self-Renewal. Cell Rep.

Brody, T., Stivers, C., Nagle, J. and Odenwald, W. F. (2002). Identification of novel Drosophila neural precursor genes using a differential embryonic head cDNA screen. Mech Dev 113, 4159.

Cheah, P. Y., Chia, W. and Yang, X. (2000). Jumeaux, a novel Drosophila winged-helix family protein, is required for generating asymmetric sibling neuronal cell fates. Development 127, 3325-35.

De Graeve, F., Jagla, T., Daponte, J. P., Rickert, C., Dastugue, B., Urban, J. and Jagla, K. (2004). The ladybird homeobox genes are essential for the specification of a subpopulation of neural cells. Dev Biol 270, 122-34.

Dorfman, R. and Shilo, B. Z. (2001). Biphasic activation of the BMP pathway patterns the Drosophila embryonic dorsal region. Development 128, 965-72.

Eade, K. T. and Allan, D. W. (2009). Neuronal phenotype in the mature nervous system is maintained by persistent retrograde bone morphogenetic protein signaling. J Neurosci 29 , 3852-64. 
Gabilondo, H., Losada-Perez, M., del Saz, D., Molina, I., Leon, Y., Canal, I., Torroja, L. and Benito-Sipos, J. (2011). A targeted genetic screen identifies crucial players in the specification of the Drosophila abdominal Capaergic neurons. Mech Dev 128, 208-21.

Haerry, T. E., Khalsa, O., O'Connor, M. B. and Wharton, K. A. (1998). Synergistic signaling by two BMP ligands through the SAX and TKV receptors controls wing growth and patterning in Drosophila. Development 125, 3977-87.

Hamanaka, Y., Park, D., Yin, P., Annangudi, S. P., Edwards, T. N., Sweedler, J., Meinertzhagen, I. A. and Taghert, P. H. (2010). Transcriptional orchestration of the regulated secretory pathway in neurons by the bHLH protein DIMM. Curr Biol 20, 9-18.

Hewes, R. S., Park, D., Gauthier, S. A., Schaefer, A. M. and Taghert, P. H. (2003). The bHLH protein Dimmed controls neuroendocrine cell differentiation in Drosophila. Development 130, 1771-81.

Hohenstein, P. and Hastie, N. D. (2006). The many facets of the Wilms' tumour gene, WT1. Hum Mol Genet 15 Spec No 2, R196-201.

Kanai, M. I., Okabe, M. and Hiromi, Y. (2005). seven-up Controls switching of transcription factors that specify temporal identities of Drosophila neuroblasts. Dev Cell 8, 203-13.

Kaspar, M., Schneider, M., Chia, W. and Klein, T. (2008). Klumpfuss is involved in the determination of sensory organ precursors in Drosophila. Dev Biol 324, 177-91.

Keshishian, H. and Kim, Y. S. (2004). Orchestrating development and function: retrograde BMP signaling in the Drosophila nervous system. Trends Neurosci 27, 143-7.

Klein, T. and Campos-Ortega, J. A. (1997). klumpfuss, a Drosophila gene encoding a member of the EGR family of transcription factors, is involved in bristle and leg development.

Development 124, 3123-34.

Losada-Perez, M., Gabilondo, H., del Saz, D., Baumgardt, M., Molina, I., Leon, Y., Monedero, I., Diaz-Benjumea, F., Torroja, L. and Benito-Sipos, J. (2010). Lineage-unrelated neurons generated in different temporal windows and expressing different combinatorial codes can converge in the activation of the same terminal differentiation gene. Mech Dev 127, 458-71. Lundgren, J., Masson, P., Mirzaei, Z. and Young, P. (2005). Identification and characterization of a Drosophila proteasome regulatory network. Mol Cell Biol 25, 4662-75.

Marques, G., Haerry, T. E., Crotty, M. L., Xue, M., Zhang, B. and O'Connor, M. B. (2003). Retrograde $\mathrm{Gbb}$ signaling through the Bmp type 2 receptor wishful thinking regulates systemic FMRFa expression in Drosophila. Development 130, 5457-70.

McDonald, J. A., Fujioka, M., Odden, J. P., Jaynes, J. B. and Doe, C. Q. (2003). Specification of motoneuron fate in Drosophila: integration of positive and negative transcription factor inputs by a minimal eve enhancer. J Neurobio/ 57, 193-203.

Mellerick, D. M., Kassis, J. A., Zhang, S. D. and Odenwald, W. F. (1992). castor encodes a novel zinc finger protein required for the development of a subset of CNS neurons in Drosophila.

Neuron 9, 789-803.

Miguel-Aliaga, I. and Thor, S. (2004). Segment-specific prevention of pioneer neuron apoptosis by cell-autonomous, postmitotic Hox gene activity. Development 131, 6093-105.

O'Keefe, D. D., Thor, S. and Thomas, J. B. (1998). Function and specificity of LIM domains in Drosophila nervous system and wing development. Development 125, 3915-23.

Park, D., Han, M., Kim, Y. C., Han, K. A. and Taghert, P. H. (2004). Ap-let neurons--a peptidergic circuit potentially controlling ecdysial behavior in Drosophila. Dev Biol 269, 95-108. Park, D., Veenstra, J. A., Park, J. H. and Taghert, P. H. (2008). Mapping peptidergic cells in Drosophila: where DIMM fits in. PLoS One 3, e1896.

Roberts, S. G. (2005). Transcriptional regulation by WT1 in development. Curr Opin Genet Dev 15, 542-7.

Suster, M. L., Martin, J. R., Sung, C. and Robinow, S. (2003). Targeted expression of tetanus toxin reveals sets of neurons involved in larval locomotion in Drosophila. J Neurobiol 55, 23346. 
Tanimoto, H., Itoh, S., ten Dijke, P. and Tabata, T. (2000). Hedgehog creates a gradient of DPP activity in Drosophila wing imaginal discs. Mol Cell 5, 59-71.

Terriente Felix, J., Magarinos, M. and Diaz-Benjumea, F. J. (2007). Nab controls the activity of the zinc-finger transcription factors Squeeze and Rotund in Drosophila development.

Development 134, 1845-52.

Wagner, N., Wagner, K. D., Hammes, A., Kirschner, K. M., Vidal, V. P., Schedl, A. and Scholz, H. (2005). A splice variant of the Wilms' tumour suppressor Wt1 is required for normal development of the olfactory system. Development 132, 1327-36.

Xiao, Q., Komori, H. and Lee, C. Y. (2012). klumpfuss distinguishes stem cells from progenitor cells during asymmetric neuroblast division. Development 139, 2670-80.

Yang, X., Bahri, S., Klein, T. and Chia, W. (1997). Klumpfuss, a putative Drosophila zinc finger transcription factor, acts to differentiate between the identities of two secondary precursor cells within one neuroblast lineage. Genes Dev 11, 1396-408. 


\section{Figure 1}
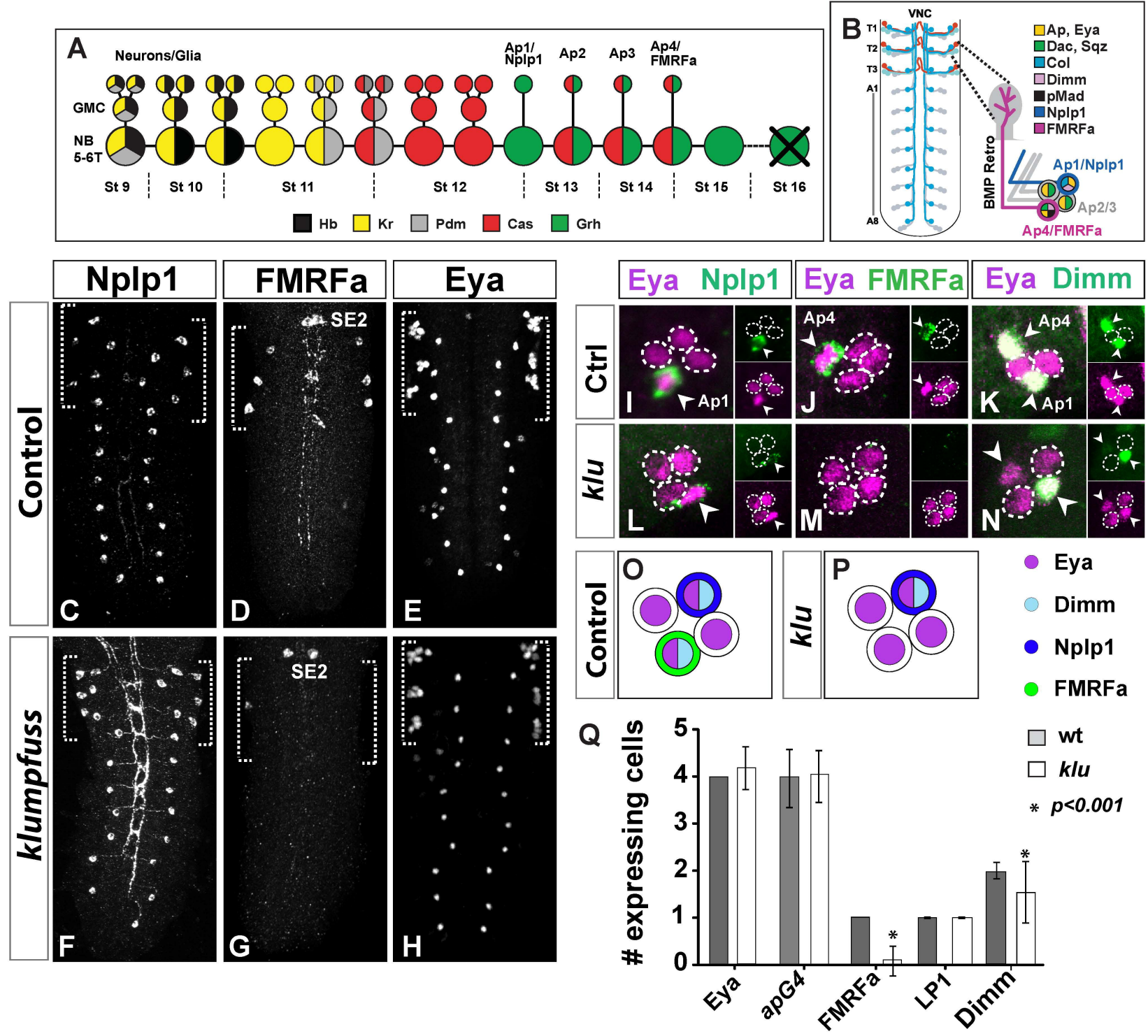


\section{Figure 2}
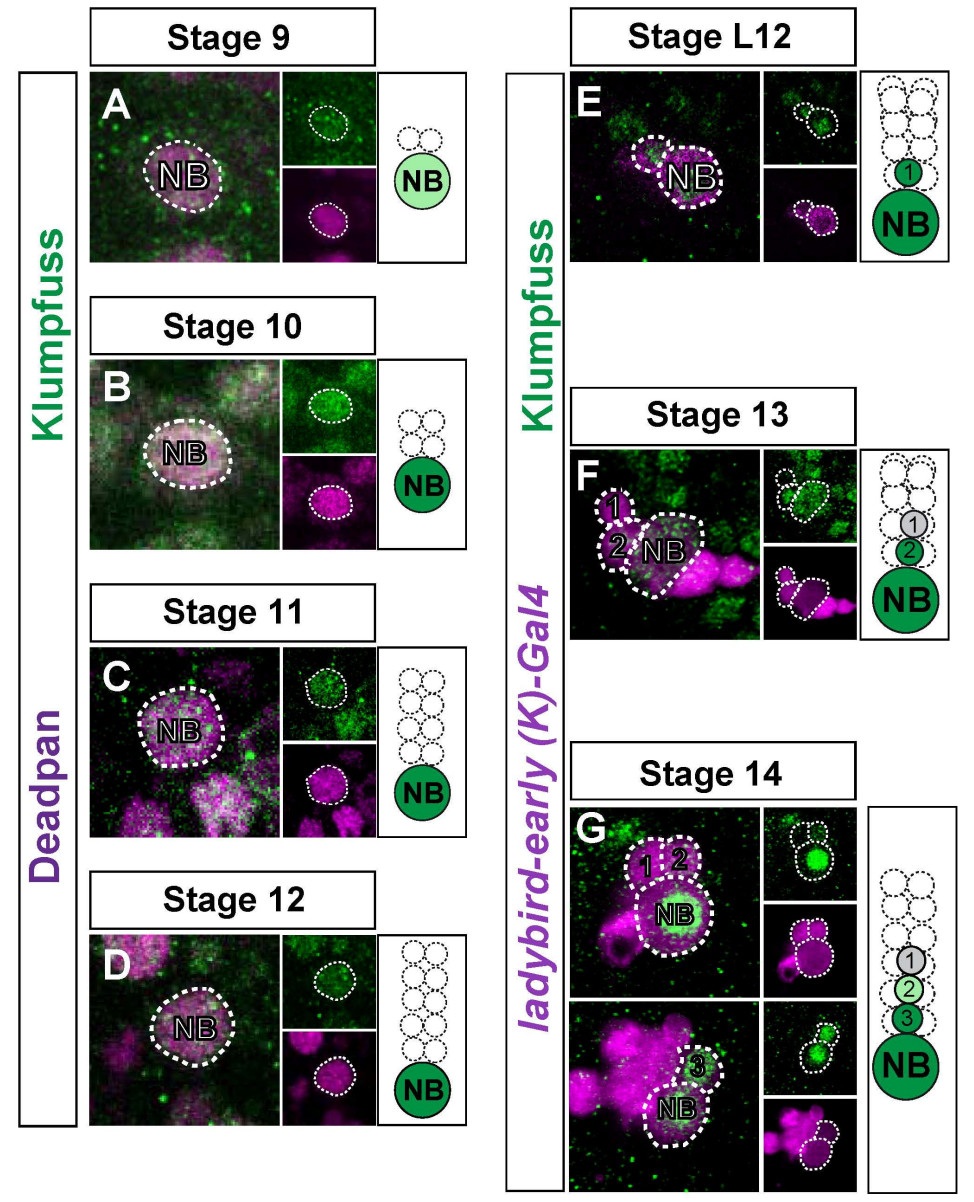

L

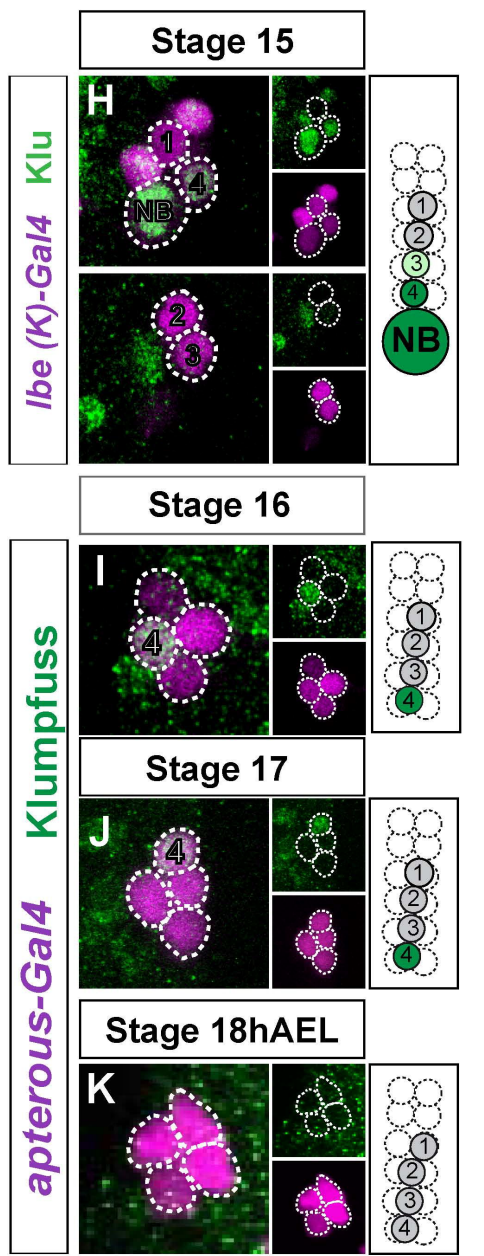

Klu +

Klu -

$1=$ Ap 1

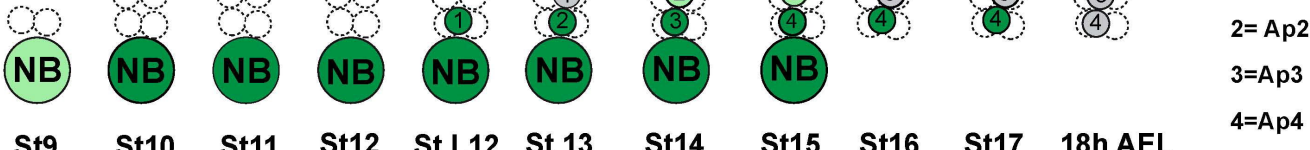

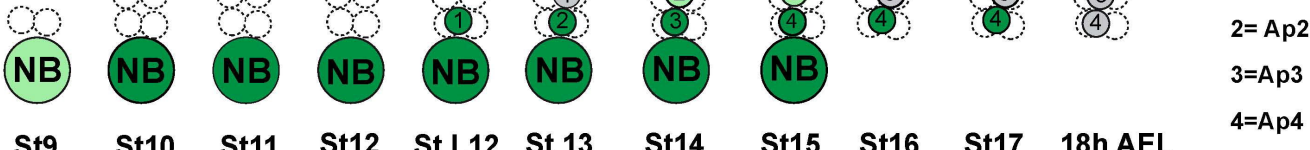

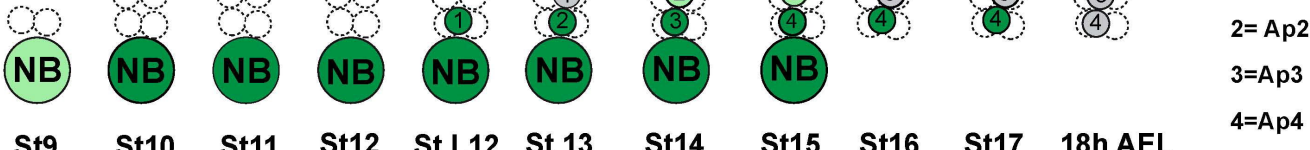




\section{Figure 3}

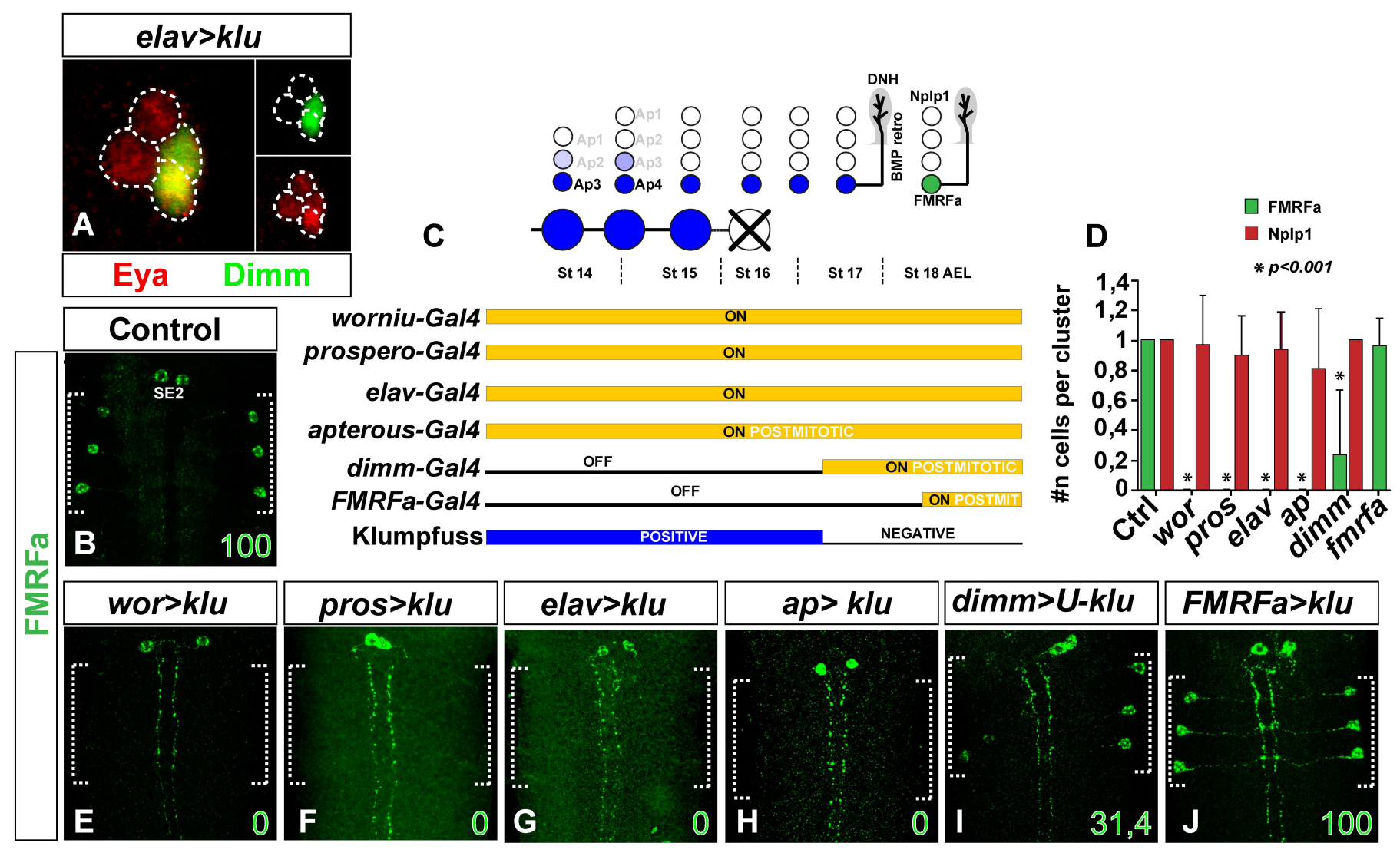




\section{Figure 4}
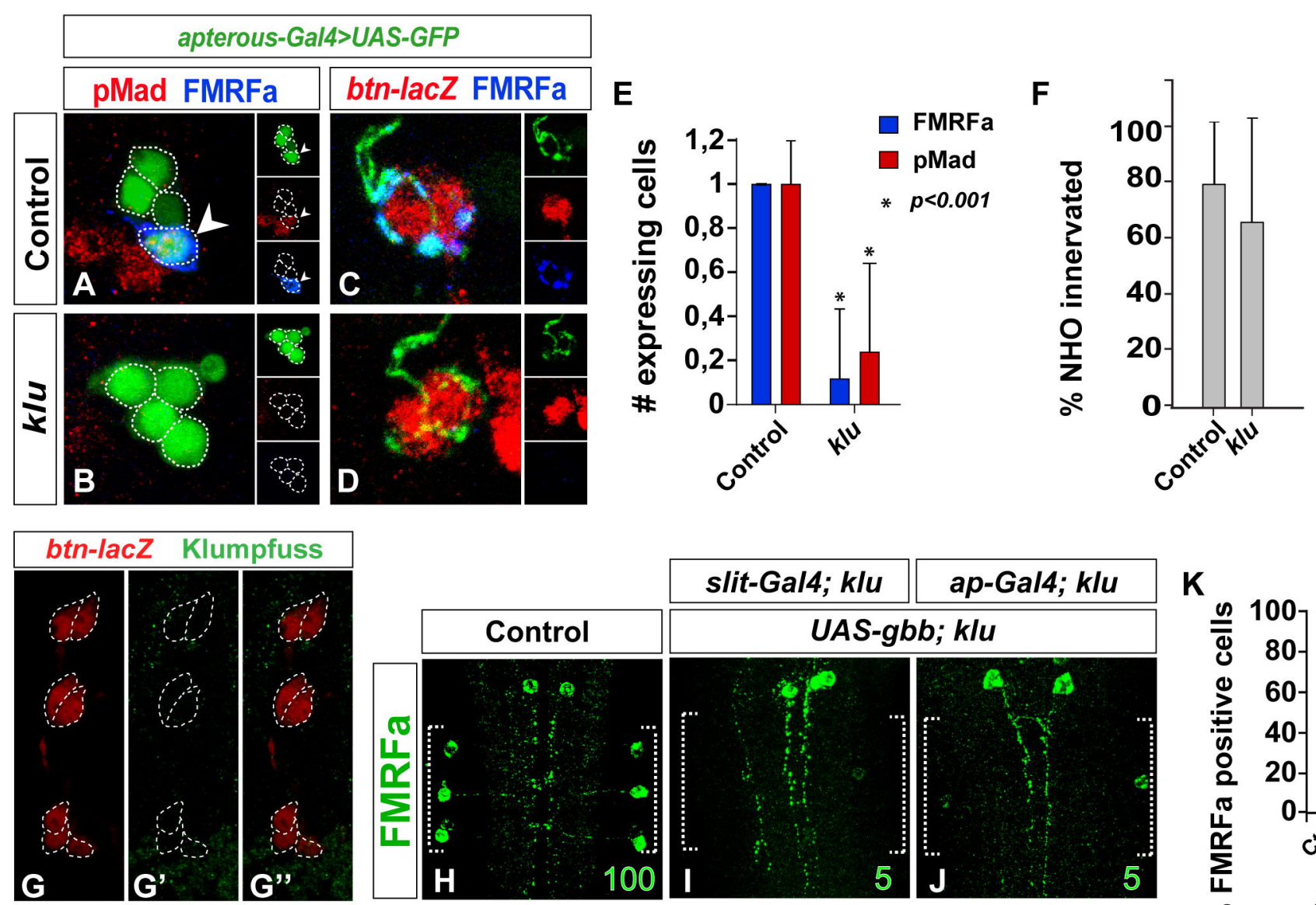

K

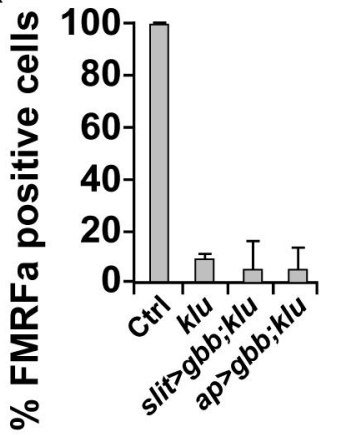




\section{Figure 5}
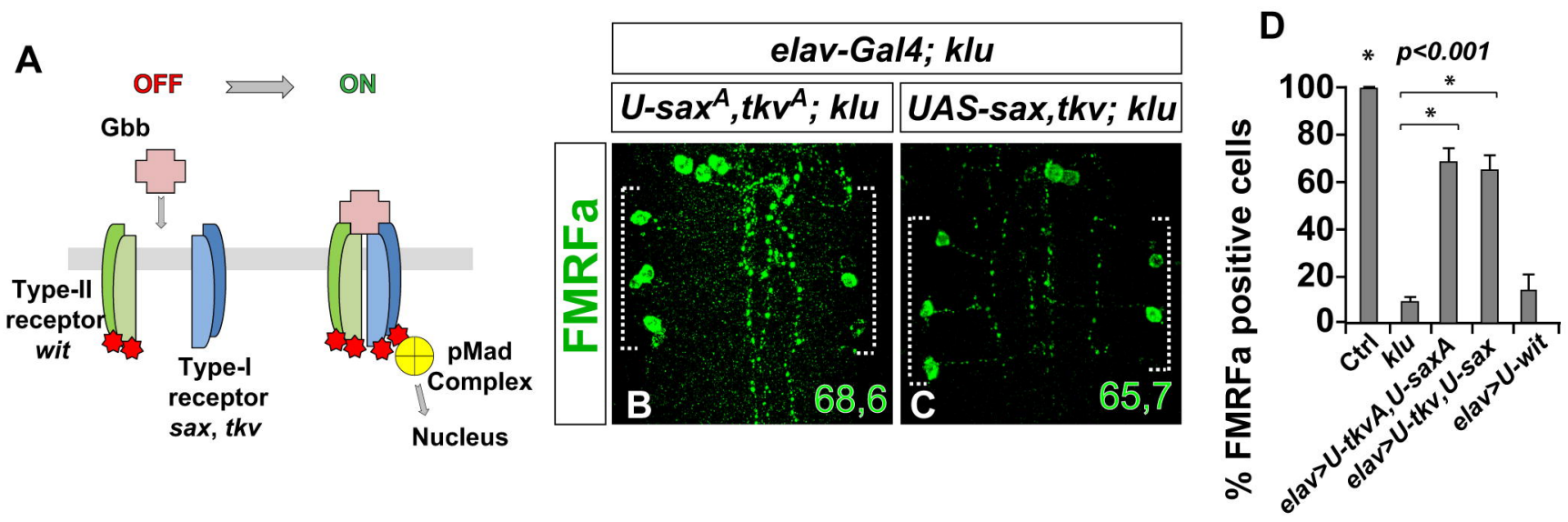